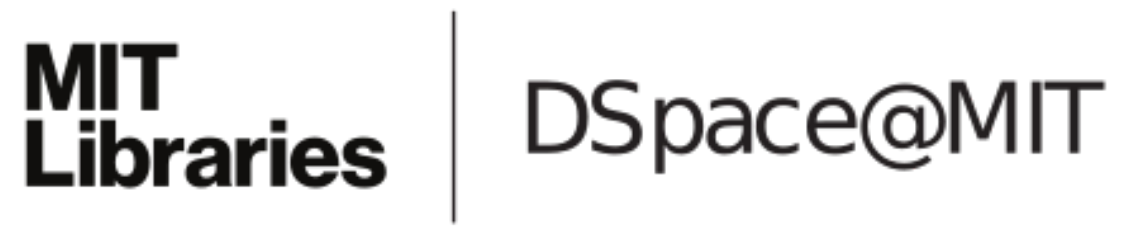

\author{
MIT Open Access Articles
}

\section{Prospects and challenges in the electromagnetic follow-up of LIGO-Virgo gravitational wave transients}

The MIT Faculty has made this article openly available. Please share how this access benefits you. Your story matters.

Citation: Vitale, Salvatore. "Prospects and Challenges in the Electromagnetic Follow-up of LIGO-Virgo Gravitational Wave Transients." Proceedings of SPIE--the Society of Photo-Optical Instrumentation Engineers, SPIE, 2014. (c) 2014 SPIE

As Published: http://dx.doi.org/10.1117/12.2057142

Publisher: SPIE

Persistent URL: http://hdl.handle.net/1721.1/109099

Version: Final published version: final published article, as it appeared in a journal, conference proceedings, or other formally published context

Terms of Use: Article is made available in accordance with the publisher's policy and may be subject to US copyright law. Please refer to the publisher's site for terms of use. 


\title{
Prospects and challenges in the electromagnetic follow-up of LIGO-Virgo gravitational wave transients
}

\author{
Salvatore Vitale ${ }^{a}$ for the LIGO Scientific Collaboration and the Virgo Collaboration \\ ${ }^{a}$ LIGO Laboratory, Massachusetts Institute of Technology, 02139 Cambridge, MA, USA
}

\begin{abstract}
The kilometer-scale ground based gravitational wave (GW) detectors, LIGO and Virgo, are being upgraded to their advanced configurations. We expect the two LIGO observatories to undertake a 3 month science run in 2015 with a limited sensitivity. Virgo should come online in 2016, and join LIGO for a 6 month science run. Through a sequence of science runs and commissioning periods, the final sensitivity should be reached by $\sim 2019$. LIGO and Virgo are expected to deliver the first direct detection of gravitational wave transients in the next few years. Most of the known sources of GWs targeted by LIGO and Virgo will likely be luminous in the electromagnetic (EM) spectrum as well. Compact binary coalescences are thought to be progenitors of short gamma-ray bursts, while long gamma-ray bursts are likely to be associated with core collapse supernova. A joint detection of gravitational and EM radiation may help confirm these associations, and expand our understanding of those astrophysical systems. Due to the transient nature, a search for the EM counterparts to GW events should be done with the shortest latency. In this paper we describe the EM follow-up program of Advanced LIGO and Virgo, from the search for GWs to the production of sky maps. Furthermore, we quantify the expected sky localization errors in the first two years of operation of the advanced detectors network.
\end{abstract}

\section{INTRODUCTION}

The advanced version of the ground based gravitational wave observatories LIGO$^{1}$ will start collecting data in 2015, followed shortly after by Advanced Virgo. ${ }^{2}$ Through a sequence of commissioning periods, they will reach their design sensitivity at the end of this decade, when they will probe a volume of the Universe a factor of $10^{3}$ larger than the initial detectors. The Japanese detector KAGRA, ${ }^{3}$ and LIGO-India ${ }^{4}$ are also expected to join this global network in the early 2020s, further increasing the sensitivity, and dramatically improving sky localization of gravitational wave (GW) sources. Ground based detectors are thus expected to make the first direct detection of gravitational radiation, and start gravitational wave astronomy.

Beside the obvious interest that a direct GW detection deserves on its own, there is much interest in the possibility of joint electromagnetic - gravitational detections. In fact GW detectors target several classes of astrophysical sources, some of which are also expected to be luminous in the electromagnetic (EM) spectrum. ${ }^{5-8}$

The most promising sources of GWs are compact binary systems (CBC) consisting of neutron stars and/or black holes, which are also the best theoretically understood. Loss of energy through gravitational wave emission makes the orbit to shrink (inspiral phase), until the two objects merge to form a single black hole. This releases the excess of energy "ringing-down" to equilibrium. The inspiral part can be described well using Post-Newtonian theory (see. ${ }^{9}$ for a review), while effective one body (EOB) models ${ }^{10}$ and Inspiral-Merger-Ringdown (IMR) phenomenological models ${ }^{11}$ can describe the whole waveform. During the last few seconds to minutes of the CBC's life the GW signal they emit will be in the LIGO-Virgo frequency band.

Compact binaries containing at least one neutron star are believed to power short $(\lesssim$ seconds) and beamed gamma-ray bursts (SGRBs). ${ }^{12-14} \mathrm{~A}$ review of the possible counterparts to CBC mergers, together with an assessment of their detectability, can be found in. ${ }^{15}$ Off-axis afterglows are also produced: X-ray and optical afterglows ${ }^{16,17}$ will peak hours to days after the merger, whereas radio afterglow emission ${ }^{17}$ will last weeks to years. Optical afterglows will fade with a power law $t^{-\alpha}$ with $\alpha$ between 1 and 1.5. One hour (day) after the SGRB trigger the apparent optical magnitude would be between 12 and 20 for CBCs at $200 \mathrm{Mpc}(50 \mathrm{Mpc}){ }^{18}$ Finally, CBC should be linked to isotropic kilonova emission, ${ }^{19,20}$ powered by the decay of heavy elements

e-mail: salvatore.vitale@ligo.org

Observatory Operations: Strategies, Processes, and Systems V, edited by Alison B. Peck, Chris R. Benn, Robert L. Seaman, Proc. of SPIE Vol. 9080, 908000 @ 2014 SPIE CCC code: $0277-786 \mathrm{X} / 14 / \$ 18 \cdot$ doi: $10.1117 / 12.2057142$ 
produced from the tidally ejected neutron-rich material, through r-process nucleosynthesis. Kilonavae signals will be dimmer, with peak luminosity, $\sim 1$ day after merger, estimated at magnitude smaller than 20 for a source at $200 \mathrm{Mpc}$, see e.g. ${ }^{20,21}$

Beside compact binaries, other astrophysical objects are expected to emit gravitational radiation. Corecollapse supernovae are likely to produce GWs measurable with LIGO and Virgo. ${ }^{22}$ These sources are less well understood than CBCs, and so are the waveform they emit and the luminosity in the gravitational wave band. While it is believed that Advanced LIGO and Virgo will be sensitive to core-collapse supernovae from our galaxy, ${ }^{22}$ whether signals from nearby galaxies are detectable depends on the mechanism that gives rise to the GW emission. Core-collapse supernovae are also expected to emit X-ray flashes ${ }^{23,24}$ in some cases long gamma-ray bursts, ${ }^{25}$ fading with a power law similar to SGRBs, and a copious amount of detectable neutrinos. ${ }^{26}$ Neutrinos can provide an independent and equally prompt trigger for such events, as they are monitored by the worldwide network of neutrino detectors. Other mechanisms have been proposed which could generate both gravitational and electromagnetic radiation, e.g. cosmic string cusps. ${ }^{27-29}$ The LIGO and Virgo detectors will also search for unmodeled short-duration signals, potentially produced by unknown sources.

Due to the transient nature of most EM counterparts, one would like to reduce the latency to a minimum, and point telescopes as soon as possible after a GW candidate has been identified. EM facilities will have to image large sky areas (initially hundreds of square degrees) while searching for dim EM signals. Furthermore, tracking light curves may require several days or weeks of observations after the GW trigger. A successful EM follow-up program will thus require three main ingredients:

- Fast detection and validation of gravitational wave candidates;

- Production of sky maps;

- Alert sent to the EM community.

During the last joint science run of Initial LIGO and Virgo (2009-2010), an end-to-end EM follow-up program has been put in place and tested successfully. Online GW searches were performed for both CBC and unmodeled bursts, and alerts to telescopes were sent out with a latency of $\sim 30$ minutes. The EM follow-up program of Initial LIGO and Virgo and its results have been fully discussed elsewhere. ${ }^{30-33}$ The LIGO-Virgo Collaborations expect to hold a similar program in the advanced detector era and have recently released a memorandum of understanding (MOU) call for the joint GW-EM follow-up program. The MOU call was very well received, and several tens of EM facilities, covering the whole EM spectrum, have expressed their interest. More details about the MOU call can be found in. ${ }^{34,35}$

When first turned on, the Advanced detectors will have a sensitivity only slightly better than their initial counterparts. Design sensitivity will be achieved through a series of science (i.e. data collecting) and commissioning periods, which should happen in the the next 4-5 years. A tentative road-map, reflecting our best guess of the commissioning operations, assumes there will be 4 science periods before 2020 , with increasingly improved sensitivity and longer duration: ${ }^{36}$

- (2015) A 3 month run with the two LIGO detectors operating at an initial strain sensitivity of $\sim 10^{-23} \mathrm{~Hz}^{-1 / 2}$, corresponding to a binary neutron star (BNS) range * of $40-80 \mathrm{Mpc}$. Virgo may join the run with a reduced range of $\sim 20 \mathrm{Mpc}$.

- (2016-17) A 6 month run with the LIGO detectors at a $80-120 \mathrm{Mpc}$ range and Virgo at 20 - $60 \mathrm{Mpc}$

- (2017-18) A 9 month run with the LIGO detectors at a 120 - 170 Mpc range and Virgo at $60-85$ Mpc

- $(2019+)$ The two LIGO observatories at full sensitivity $(200 \mathrm{Mpc})$ and Virgo at $\sim 65 \mathrm{Mpc}$.

On Fig. 1 (from ${ }^{36}$ ) we show the expected evolution of the detector sensitivity for LIGO (left panel) and Virgo (right panel) from 2015 to $\sim 2020$, together with the BNS-optimized noise spectrum. ${ }^{37}$

${ }^{*}$ This is the volume and orientation averaged distance at which a $(1.4,1.4) M_{\odot}$ BNS would produces a singleinterferometer signal-to-noise ratio (SNR) of 8, usually considered the threshold for detection. 

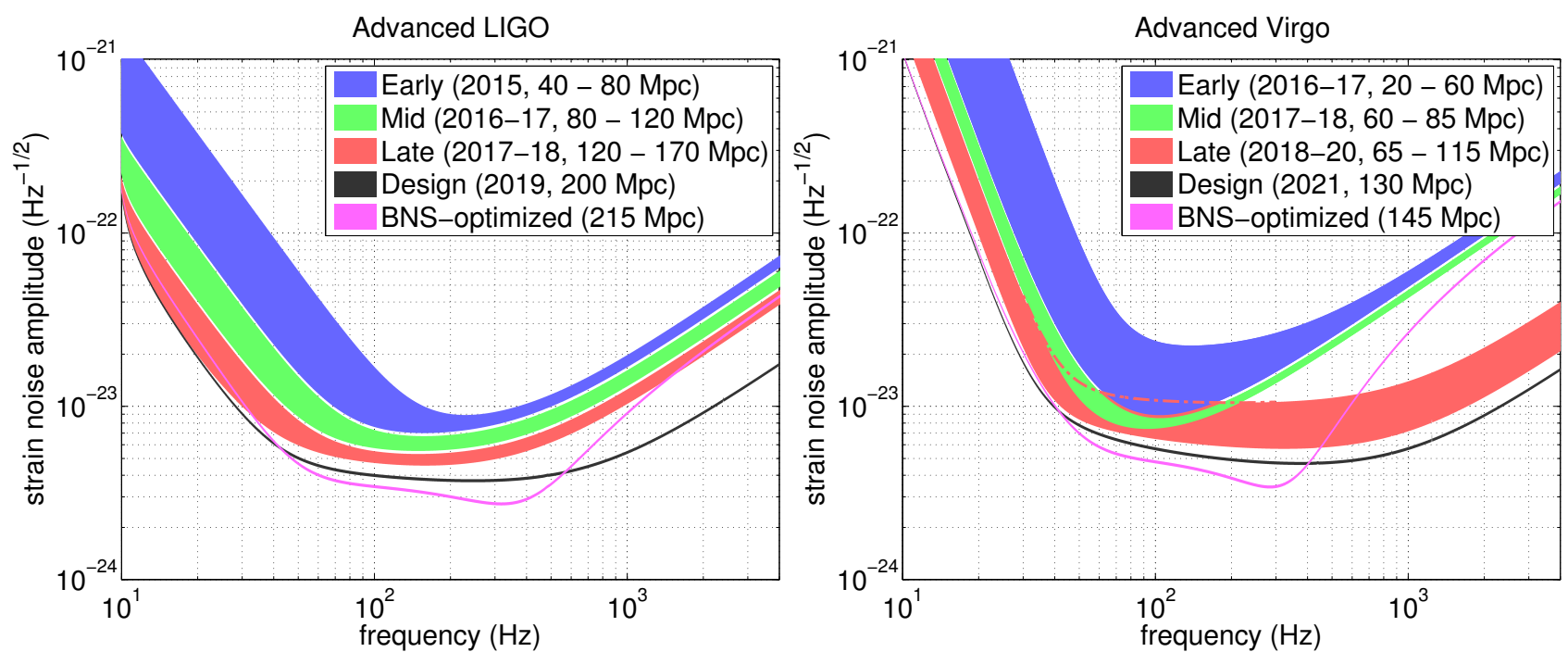

Figure 1. Expected evolution of the noise strain sensitivity for Advanced LIGO (left) and Virgo (right). Once design sensitivity is reached, the hardware configuration can be slightly modified to increase the BNS range (BNS-optimized curves)

In this paper we describe the EM program of LIGO and Virgo, identify and quantify the main sources of latency, and the way information will be transmitted to the partner telescopes. The rest of this paper is organized as follows: in section 2 we describe the low-latency search for GW transients in LIGO-Virgo data, the algorithms uses to quickly produce sky maps, and the way information about GW triggers will be communicated to EM facilities. In section 3 we review the expected progress of sky localization capabilities in the Advanced LIGO-Virgo era, focusing in particular on the first 2 years (2015-2016).

\section{FROM TRIGGER GENERATION TO EM OBSERVATION}

In this section we summarize the main steps from data taking to sky map generation and EM observations, and their expected latencies. This is also represented in the schematic flow chart in Fig. 2.

\subsection{Candidate Selection}

The LIGO-Virgo Collaborations expects to run two online CBC searches, which will help cross checking for consistency: GSTLAL and MBTA.

The Multi-Band Template Analysis (MBTA ${ }^{38}$ pipeline has already been successfully used in the initial detector era to generate triggers for the EM follow-up program. ${ }^{30-33}$ MBTA is a matched filter algorithm ${ }^{39}$ which uses a bank of templates (created before the analysis, and updated regularly) to search for potential $\mathrm{CBC}$ events in each interferometer's data. The search is done by filtering the data in the frequency domain, and using two frequency bands to reduce the sampling rate needed for the low-frequency part of the templates. Triggers for each instrument are clustered and used to search for coincidences across the network. Candidate events must have consistent arrival time in each detector and chirp mass ${ }^{\dagger}$. GSTLAL ${ }^{40}$ has been recently developed, and thus not used on previous science runs. It has been, however, extensively tested on simulated GW signals and in engineering runs. Unlike MBTA, GSTLAL filters the data directly in the time domain, using an orthogonal template bank and singular-value decomposition. ${ }^{41,42}$ GSTLAL can run with zero-latency and might even produce early warnings, i.e. potentially recognize the presence of a $\mathrm{GW}$ in the data before the actual coalescence $^{40} \ddagger$. Similar to MBTA, consistent timing and masses across the network are required to consider a trigger as a candidate event.

\footnotetext{
${ }^{\dagger}$ defined as $\mathcal{M} \equiv\left[m_{1}^{3} m_{2}^{3} /\left(m_{1}+m_{2}\right)\right]^{1 / 5}, m_{1}$ and $m_{2}$ being the individual masses of the two compact objects.

${ }^{\ddagger}$ One may hope that early warnings might be used to point telescopes before the CBC coalesces. Unfortunately ref. ${ }^{40}$ shows how this would typically result in prohibitively large sky maps.
} 
If the noise in the interferometers were Gaussian, the matched filter signal-to-noise ratio would be sufficient to assess the statistical significance of a candidate event and its false alarm rate (FAR). However non-Gaussian noise artifacts (glitches), which were present in the initial detectors, are likely to also affect the advanced detectors. To help discriminate between glitches and real GWs, candidate events will thus be required to pass a $\chi^{243}$ test. The FAR of the surviving candidate events is then explicitly calculated. GSTLAL assigns a false alarm probability by using the SNR and $\chi^{2}$ across the network, ${ }^{44}$ while MBTA uses a Poisson point estimate. ${ }^{45}$ CoherentWave Burst (cWB) ${ }^{46}$ will be used to carry the online search for unmodeled short-lived GWs (bursts), while a second pipeline might be run to assure an appropriate cross checking. cWB performs a time-frequency analysis of the data in the wavelet domain, and has been used to carry burst searches in the initial detector era. ${ }^{47,48}$ Unlike the CBC algorithms discussed above, cWB implements a coherent search, i.e. it uses data from all the detectors from the start of the analysis, instead of first creating single-instrument triggers.

The FAR of cWB candidate events is calculated using the time-shift method. ${ }^{47}$ In this method, artificial time shifts,

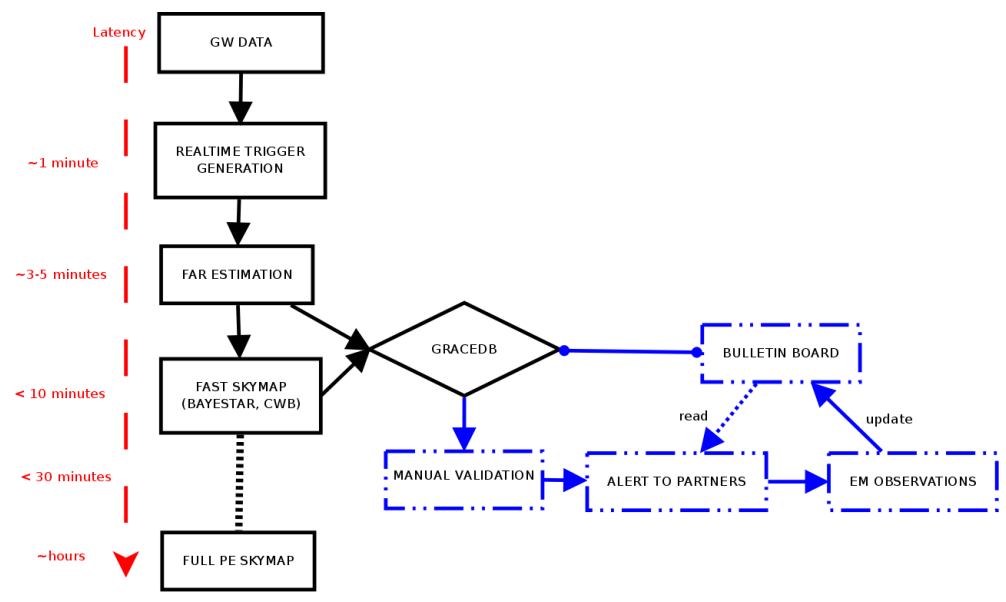

Figure 2. Schematic flowchart of the online analysis, from trigger generation to EM follow-up. We are assuming that the GW candidate was significant enough to require an EM follow-up. In the opposite case the blue dashed boxes would not be executed. The cumulative latency since data acquisition is given on the left of the plot. The LIGO-Virgo Collaborations is considering automatizing the validation of events, before the alert is sent. However this will probably not happen before the first few detections are made. We are thus considering manual validation in this flowchart. larger than the time of flight between detectors, are applied to the data of one or more detectors. The time-shifted data is reanalized, providing the background distribution of coincident triggers in absence of real GWs. The background is continuously evaluated, to monitor changes of behavior in the interferometers. The latency from data acquisition to candidates selection is $\lesssim 5$ minutes for all of the above algorithms.

In the initial detector era there was an extra step involving human validation before significant events could be considered for EM follow-up. Scientists in the control rooms of LIGO and Virgo would check the quality of the data and the overall condition of the running instruments around the time of the trigger, both on the GW and the auxiliary channels. The latency introduced by the manual vetting was typically $\sim 10-20$ minutes, and thus represented a significant fraction of the total latency. The LIGO-Virgo Collaborations is working toward providing data quality information in low-latency in the advanced detector era and fully automating the validation process. However it is likely this will not happen from the very beginning of the GW follow-up program.

\subsection{Sky localization}

Triggers surviving the final validation are assigned a unique ID and added, together with some relevant information (SNR, time, chirp mass for CBC events, data quality, etc.), to the gravitational-wave candidate event database (GraceDB) ${ }^{49}$ a queryable web service that collects candidate GW events. Whenever a new event is added, GraceDB sends an alert (LVAlert ${ }^{50}$ ) to LIGO-Virgo users (humans or robots) which are listening to a particular channel (e.g. CBC, unmodeled bursts, etc). In this subsection we will describe a specific class of listeners: programs that reconstruct the sky position of the candidate event.

The antenna pattern of an intereferometric gravitational wave detector is maximum directly overhead and underneath the plane spanned by the two arms, and smoothly decreases until it reaches the minimum in that 
plane. LIGO and Virgo are thus sensitive to all sky directions, with poor angular resolution. ${ }^{39}$ At the lowest order, the sky position of a GW trigger can be calculated using time triangulation. ${ }^{51}$ In this approximation, the timing error at each instrument is given by the simple expression $\sigma_{t}=\left(2 \pi \rho \sigma_{f}\right)^{-1}$, where $\rho$ is the SNR of the template and $\sigma_{f}$ is the effective bandwidth of the detector. ${ }^{51}$ Time triangulation has the advantage of being very fast, as it does not require much computation, and it was used to produce CBC sky maps for the EM follow-up program in the initial detector era. However, it only uses timing information, and one may expect that more reliable sky maps can be built by also taking into account the amplitude of the GW across the network.

BAYESTAR $^{52}$ is a recently developed algorithm that can estimate the position of CBC triggers, using timing, phase, and amplitude consistency, with only a short latency, typically $\lesssim 1$ minute. In order to achieve such low latencies, BAYESTAR makes some simplifying assumptions about the signals. In particular, it assumes that the objects do not carry significant spins, which should be a good approximation for BNS. ${ }^{53}$ It further assumes that the binary component masses have the values estimated by the detection pipeline, which results in a significant speed up ${ }^{\S}$. The marginalized sky posterior is thus obtained by numerically integrating the full posterior distribution.

Skymaps of short GW bursts emitted by other kinds of astrophysical sources are generated using cWB, with typical latencies of $\lesssim 1$ minute. cWB grids the sky and calculates a network likelihood statistic by combining data of all interferometers taking part in the analysis. The sky pixels are then ranked by decreasing likelihood, and normalized to yield a 2-D posterior distribution.

Both cWB and BAYESTAR will produce a FITS file with an ordered list of pixels and the resulting sky map, and update the GraceDB entry for the event.

\subsection{EM Observation and updates to GraceDB}

Once the sky map is available on GraceDB, a robot checks if the FAR is below the threshold LIGO and Virgo fixed to proceed with EM follow-up. For triggers which pass this criterion, an alert will be sent to the partner EM facilities. A VOEvent-formatted notice will be circulated through a private version of GCN, ${ }^{54}$ also containing a link to a FITS file sky map. Each EM facility may decide to further downselect the events to follow, if the threshold FAR fixed by the LIGO-Virgo Collaborations resulted in impractically frequent observations.

As detailed below, especially in the first few years the sky error regions associated with GW will be much larger than the field of view of most optical telescopes, typically spanning hundreds of square degrees. Furthermore, the expected luminosity of most EM counterparts spans several order of magnitude, in several EM bands. In facilitating the EM search, the LIGO-Virgo Collaborations might provide a platform for communication among all interested parties in the form of a Bulletin Board attached to the GW event database GraceDB. EM partners would thus be able to upload to the Bulletin Board the area that they surveyed and any relevant information about the observations.

\subsection{Refined sky maps and subsequential updates}

Together with the fast sky localization codes described above, LALInference, a Bayesian parameter estimation algorithm for CBC signals, will also be run. LALInference has been extensively used both on real ${ }^{55}$ and simulated data. Unlike BAYESTAR, it does not assume that masses are known, and instead performs a full Markov Chain Monte Carlo exploration of the parameter space. Runs take $\sim$ hours to days to complete, depending on the complexity of the model (e.g. whether spins are taken into account or not). Together with a refined sky map, LALInference also provides estimates of all parameters on which CBC depends, e.g. luminosity distance, orbital inclination, spins (if considered). The updated version of the sky map will be uploaded to GraceDB once the full parameter estimation analysis is done. Given the longer latency, these sky maps may not be available for EM searches of the early afterglow emission, but can be used to help the search for kilonovae emission, and possibly re-evaluate the significance of EM counterparts found in the early stages of the follow-up.

\footnotetext{
${ }^{\S}$ A new waveform must be calculated every time the intrinsic parameters (masses and spins) are varied. By neglecting spins and fixing the mass parameters one needs to only calculate one waveform and just project it to the desired sky positions and orientations.
} 
Days or weeks after the event, the LIGO-Virgo Collaborations may perform an offline calibration of the instruments, and revise the calibration used in the online search. Even though calibration errors of the level encountered in the initial detector era should not significantly affect the sky localization of BNS, ${ }^{56,57}$ an updated version of the sky map which uses the new calibration may be uploaded to GraceDB.

\section{EXPECTED SKY LOCALIZATION CAPABILITIES}

The size and shape of typical sky maps will vary significantly over the next few years, depending on the number of detectors and their relative sensitivities, as well as the morphology of the GW signal.

As mentioned above, in ref. ${ }^{36}$ we explored the plausible evolution of the GW network from 2015 to 2020, when both LIGO and Virgo should have reached their design sensitivities. Using time triangulation and the expected coalescence rates of compact binaries, ${ }^{58}$ it also quantified the range of the network, gave ranges for the possible number of detections, and typical sky localization precision. We report Table 1 of ${ }^{36}$ here below.

\begin{tabular}{|c|c|c|c|c|c|c|c|c|}
\hline & Estimated & \multicolumn{2}{|c|}{$E_{\mathrm{GW}}=10^{-2} M_{\odot} c^{2}$} & \multicolumn{2}{c|}{ Number } & \multicolumn{2}{c|}{$\begin{array}{c}\text { BNS Localized } \\
\text { of BNS }\end{array}$} & \multicolumn{2}{c|}{ within } \\
& Run & \multicolumn{2}{c|}{ Burst Range $(\mathrm{Mpc})$} & \multicolumn{2}{c|}{ BNS Range (Mpc) } & of \\
Epoch & Duration & LIGO & Virgo & LIGO & Virgo & Detections & $5 \mathrm{deg}^{2}$ & $20 \mathrm{deg}^{2}$ \\
\hline 2015 & 3 months & $40-60$ & - & $40-80$ & - & $0.0004-3$ & - & - \\
$2016-17$ & 6 months & $60-75$ & $20-40$ & $80-120$ & $20-60$ & $0.006-20$ & 2 & $5-12$ \\
$2017-18$ & 9 months & $75-90$ & $40-50$ & $120-170$ & $60-85$ & $0.04-100$ & $1-2$ & $10-12$ \\
$2019+$ & (per year) & 105 & $40-80$ & 200 & $65-130$ & $0.2-200$ & $3-8$ & $8-28$ \\
$2022+$ (India) & (per year) & 105 & 80 & 200 & 130 & $0.4-400$ & 17 & 48 \\
\hline
\end{tabular}

Table 1. Summary of a plausible observing schedule, expected sensitivities, and source localization (size of the $90 \%$ confidence area) with the advanced LIGO and Virgo detectors, which will be strongly dependent on the detectors' commissioning progress. The burst ranges assume standard-candle emission of $10^{-2} M_{\odot} c^{2}$ in $\mathrm{GWs}$ at $150 \mathrm{~Hz}$ and scale as $E_{\mathrm{GW}}^{1 / 2}$. The burst and BNS ranges and the BNS localization reflect the uncertainty in the detector noise spectra shown in Fig. 1. The BNS detection numbers also account for the uncertainty in the BNS source rate density, ${ }^{58}$ and are computed assuming a false alarm rate of $10^{-2} \mathrm{yr}^{-1}$. Burst localizations are expected to be broadly similar to those for BNS systems, but will vary depending on the signal bandwidth. Localization and detection numbers assume an $80 \%$ duty cycle for each instrument. $\left(\right.$ From $^{36}$ )

From Table 1 it is clear that the fraction of well localized events will constantly increase in the next few years. Adding a LIGO detector in India will dramatically improve sky localization (several studies have assessed sky localization capabilities with future networks, both in the context of CBC and burst signals, e.g. ${ }^{59-62}$ ). For a 2 interferometer network, the time triangulation approach would lead to errors regions shaped as rings centered on the line which joins the two observatories, with corresponding error areas of hundreds to thousands of square degrees. ${ }^{58}$

More robust estimates of the typical errors for localizing BNS sources in 2015 and 2016 have been presented in $^{52}$ (see also the open data release ${ }^{63}$ ). Starting from an astrophysically motivated population of BNS, ${ }^{52}$ simulates all the steps that would be followed in real science run, which includes running detection pipelines, selecting candidate events, and building sky maps using both BAYESTAR and LALInference. The resulting picture is more optimistic than what would be obtained using time triangulation. Assuming a 3 month long 2015 run and selecting BNS with false alarm rate $(\mathrm{FAR})<10^{-2} \mathrm{yr}^{-1}$ and network SNR $>12,{ }^{52}$ shows how the searched area (i.e. the area of sky one would have to scan starting from the most likely position until the true position is found) is smaller than $500 \mathrm{deg}^{2}$ for $87 \%$ of the signals, smaller than $100 \mathrm{deg}^{2}$ for $45 \%$ of the signals, and smaller than $5 \mathrm{deg}^{2}$ for $3 \%$ of the signals, using BAYESTAR. The full parameter estimation algorithm, LALInference, obtains respectively $89 \%, 54 \%$ and $4 \%$, which shows how for the two detector network sky errors cannot be reduced much once time, amplitude and phase consistency have been taken into account. ${ }^{52}$

Typical 2015 sky error regions will consist of one or two separated islands of probability. While three modes are also possible, they will be encountered more rarely. Due to their positions and orientations, the two LIGO observatories will be most sensitive to GW sources above North America and the Indian Ocean. The sky maps of most detected events will consist of two modes, corresponding to these two regions. A typical sky map for the 2015 configuration is shown in Fig. 3 (from $^{52}$ ) 


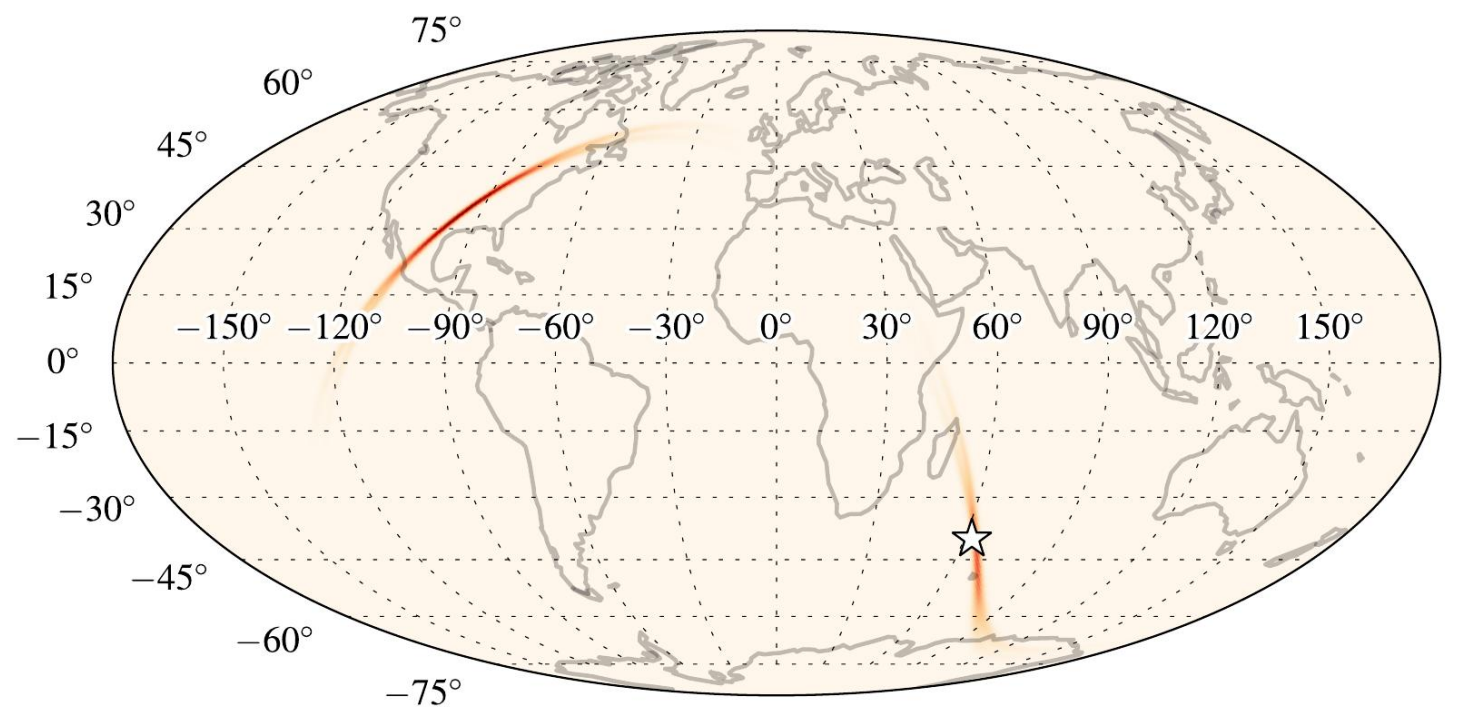

Figure 3. A representative BNS sky map for the 2015 network configuration. This is an event with a moderate network SNR of 15 . The $90 \%$ confidence region spans $700 \mathrm{deg}^{2}$ over two modes. The star represents the true position of the GW source. $\left(\right.$ From $\left.^{52}\right)$

The 2016 science run, with Virgo also online with limited sensitivity, will share some common features with the 2015 scenario. In fact, given the astrophysical distribution of the expected source distances, most events will be detected at low network SNR ?. Due to the initially large gap in sensitivity between LIGO and Virgo, this implies that the SNR in Virgo will be often below threshold, and not contribute much to sky localization. Virgo will instead help reducing the sky errors for the medium and high SNR events.

For the 2016 science run, ${ }^{52}$ finds that the searched area is smaller than $500 \mathrm{deg}^{2}$ for $82 \%$ of the signals, smaller than $100 \mathrm{deg}^{2}$ for $45 \%$ of the signals, and smaller than $5 \mathrm{deg}^{2}$ for $10 \%$ of the signals, using BAYESTAR. Using LALInference one would instead get respectively $92 \%, 70 \%$ and $20 \%$. The increased fraction of well localized events is due to the fact that for the best events Virgo will play a significant role, partially or totally breaking down the 2-detector degeneracy. For those events the sky maps will show a single mode, roughly a few tens of square degrees.

We notice that while the performances of BAYESTAR and LALInference are quite similar in the 2015 runs, LALInference seems to do generally much better in the 2016 network configuration. As we mentioned already, Virgo won't typically contribute much to the network SNR in 2016. Those detections will thus be equivalent to a two detector analysis for BAYESTAR, as BAYESTAR does not use data from detectors whose SNR is below threshold. This explains why the 2015 and 2016 performances for BAYESTAR are very similar, except for the top $\sim 30 \%$ of signals (see also Table 1 of $^{52}$ ). On the other hand, LALInference uses all data, regardless of the SNR, and can break the two detector degeneracy, at least partially. This implies that a significantly better sky map may be available for most events in the 2016 network configuration in a timescale of hours. However, ongoing projects may significantly reduce the runtime of LALInference (e.g. ${ }^{64,65}$ ), and the low-latency analysis might make use of sub-threshold data. As stressed in, ${ }^{52}$ it is thus possible that the extra latency needed to calculate better sky maps may be a limitation that can be overcome before the 2016 science run. A large-scale numerical study similar to ${ }^{52}$ may be repeated to assess sky localization capabilities in later science runs, and to complement the time-triangulation estimates given in ${ }^{36}$ and reported on Table 1.

\footnotetext{
"This is defined as the squared sum of the single interferometer SNRs
} 


\section{CONCLUSIONS}

The advanced version of the LIGO observatories is expected to start collecting data in 2015, while Virgo should come online in 2016. This network of GW detectors should deliver the first direct detection of GWs. Most GW sources, such as compact binary coalescences or core collapse supernovae, are also expected to emit in the electromagnetic band. The detection of an EM counterpart would have a huge impact on both gravitational and electromagnetic astrophysics. It will help confirming that compact binary coalescences are progenitors of short gamma-ray bursts, and core collapse supernovae are progenitors of long gamma-ray bursts. Simultaneous access to gravitational and electromagnetic data will deepen our understanding of the underlying astrophysical mechanisms, as they give complementary information: GW data will follow the motion of the mass in the source, whereas EM emission also probes the local environment. Finally, detecting an EM counterpart will increase the confidence in the GW detection. These prospects have raised interest and enthusiasm in the astrophysical community, and tens of facilities have expressed their interest in following-up GW triggers, and signed a memorandum of understanding with the LIGO-Virgo Collaborations. However, a successful EM follow-up program of GW transients will present major challenges.

Given the transient nature of most EM counterparts, GW events should be followed-up in the promptest way to increase the chances of success. GSTLAL, MBTA and cWB will search for transient GW signals in the LIGO-Virgo data, with latencies of $\sim 2$ minutes from data acquisition to identification of GW candidates. Assigning a false alarm ratio and building a sky map will add $\sim 8$ minutes to the latency. Human validation will be the largest source of latency, typically requiring $\sim 15-20$ minutes, and may eventually be dropped in favor of a fully automated process. The LIGO-Virgo Collaborations will thus be able to send alerts to EM partners with latencies of $\lesssim 30$ minutes or better.

Especially in the first few years of operation, LIGO and Virgo will not localize events on the sky with high precision. The relative orientation of the two LIGO detectors implies that typical sky maps in the 2015 science run (without Virgo) will consist of two elongated modes, $\sim 12$ hours apart. $\sim 45 \%$ of the time the source will be found imaging $100 \mathrm{deg}^{2}$ or less, while only $3 \%$ of the sources will be found imaging less than $5 \mathrm{deg}^{2}$. The presence of Virgo in 2016 run will increase the number of well localized events $\left(\sim 10-20 \%\right.$ found imaging $5 \mathrm{deg}^{2}$ or less), while for typical low signal-to-noise ratio events the sky errors will still require imaging several tens to hundreds of square degrees. Although the luminosity of expected EM counterparts depends on several unknown factors (e.g. circumburst density, jet energy), it is believed that the flux will typically be faint for sources in the range of Advanced LIGO and Virgo. The EM facilities involved in the EM follow-up program will thus be faced with the task of search for dim signals in large areas.

The next few years will be an exciting and challenging time for gravitational and electromagnetic astrophysics. The large number of facilities that will follow-up LIGO-Virgo events significantly increases the odds that, with strategic and coordinate planning, EM counterparts successfully be identified.

\section{ACKNOWLEDGMENTS}

The authors gratefully acknowledge the support of the United States National Science Foundation for the construction and operation of the LIGO Laboratory, the Science and Technology Facilities Council of the United Kingdom, the Max-Planck-Society, and the State of Niedersachsen/Germany for support of the construction and operation of the GEO 600 detector, and the Italian Istituto Nazionale di Fisica Nucleare and the French Centre National de la Recherche Scientifique for the construction and operation of the Virgo detector. The authors also gratefully acknowledge the support of the research by these agencies and by the Australian Research Council, the International Science Linkages program of the Commonwealth of Australia, the Council of Scientific and Industrial Research of India, the Istituto Nazionale di Fisica Nucleare of Italy, the Spanish Ministerio de Economía y Competitividad, the Conselleria d'Economia Hisenda i Innovació of the Govern de les Illes Balears, the Foundation for Fundamental Research on Matter supported by the Netherlands Organisation for Scientific Research, the Polish Ministry of Science and Higher Education, the FOCUS Programme of Foundation for Polish Science, the Royal Society, the Scottish Funding Council, the Scottish Universities Physics Alliance, the National Aeronautics and Space Administration, the National Research Foundation of Korea, Industry Canada and the Province of Ontario through the Ministry of Economic Development and Innovation, the National Science and

Proc. of SPIE Vol. $9149914907-8$ 
Engineering Research Council Canada, the Carnegie Trust, the Lev- erhulme Trust, the David and Lucile Packard Foundation, the Research Corporation, and the Alfred P. Sloan Foundation. This is LIGO document P1400086.

\section{REFERENCES}

[1] Harry, G. M., "Advanced LIGO: The next generation of gravitational wave detectors," Class.Quant.Grav. 27, 084006 (2010).

[2] Acernese, F. and et al., "Advanced Virgo Baseline Design," Virgo Technical report VIR-027A-09 (2013).

[3] Somiya, K., "Detector configuration of KAGRA-the Japanese cryogenic gravitational-wave detector," Classical and Quantum Gravity 29, 124007 (June 2012).

[4] Iyer, B. and et al., "Ligo-india, proposal of the consortium for indian initiative in gravitational-wave observations," (2011).

[5] Sylvestre, J., "Prospects for the Detection of Electromagnetic Counterparts to Gravitational Wave Events," ApJ 591, 1152-1156 (July 2003).

[6] Kanner, J., Huard, T. L., Márka, S., Murphy, D. C., Piscionere, J., Reed, M., and Shawhan, P., "LOOC UP: locating and observing optical counterparts to gravitational wave bursts," Classical and Quantum Gravity 25, 184034 (Sept. 2008).

[7] Stubbs, C. W., "Linking optical and infrared observations with gravitational wave sources through transient variability," Classical and Quantum Gravity 25, 184033 (Sept. 2008).

[8] Bloom, J. S., Holz, D. E., Hughes, S. A., Menou, K., Adams, A., Anderson, S. F., Becker, A., Bower, G. C., Brandt, N., Cobb, B., Cook, K., Corsi, A., Covino, S., Fox, D., Fruchter, A., Fryer, C., Grindlay, J., Hartmann, D., Haiman, Z., Kocsis, B., Jones, L., Loeb, A., Marka, S., Metzger, B., Nakar, E., Nissanke, S., Perley, D. A., Piran, T., Poznanski, D., Prince, T., Schnittman, J., Soderberg, A., Strauss, M., Shawhan, P. S., Shoemaker, D. H., Sievers, J., Stubbs, C., Tagliaferri, G., Ubertini, P., and Wozniak, P., "Astro2010 Decadal Survey Whitepaper: Coordinated Science in the Gravitational and Electromagnetic Skies," ArXiv e-prints (Feb. 2009).

[9] Blanchet, L., "Gravitational Radiation from Post-Newtonian Sources and Inspiralling Compact Binaries," Living Reviews in Relativity 9, 4 (June 2006).

[10] Buonanno, A. and Damour, T., "Effective one-body approach to general relativistic two-body dynamics," Phys. Rev. D 59, 084006 (Apr. 1999).

[11] Ajith, P., Babak, S., Chen, Y., Hewitson, M., Krishnan, B., Whelan, J. T., Brügmann, B., Diener, P., Gonzalez, J., Hannam, M., Husa, S., Koppitz, M., Pollney, D., Rezzolla, L., Santamaría, L., Sintes, A. M., Sperhake, U., and Thornburg, J., "A phenomenological template family for black-hole coalescence waveforms," Classical and Quantum Gravity 24, 689 (Oct. 2007).

[12] Nakar, E., "Short-hard gamma-ray bursts," Phys. Rept. 442, 166-236 (2007).

[13] Mészáros, P., "Gamma-ray bursts," Reports on Progress in Physics 69, 2259-2321 (Aug. 2006).

[14] Piran, T., "The physics of gamma-ray bursts," Rev. Mod. Phys. 76, 1143-1210 (Jan 2005).

[15] Metzger, B. D. and Berger, E., "What is the Most Promising Electromagnetic Counterpart of a Neutron Star Binary Merger?," ApJ 746, 48 (Feb. 2012).

[16] Coward, D. M., Gendre, B., Sutton, P. J., Howell, E. J., Regimbau, T., Laas-Bourez, M., Klotz, A., Boër, M., and Branchesi, M., "Towards an optimal search strategy of optical and gravitational wave emissions from binary neutron star coalescence," MNRAS 415, L26-L30 (July 2011).

[17] Nakar, E. and Piran, T., "Detectable radio flares following gravitational waves from mergers of binary neutron stars," Nature 478, 82-84 (Oct. 2011).

[18] Kann, D. A., Klose, S., Zhang, B., Covino, S., Butler, N. R., Malesani, D., Nakar, E., Wilson, A. C., Antonelli, L. A., Chincarini, G., Cobb, B. E., D’Avanzo, P., D’Elia, V., Della Valle, M., Ferrero, P., Fugazza, D., Gorosabel, J., Israel, G. L., Mannucci, F., Piranomonte, S., Schulze, S., Stella, L., Tagliaferri, G., and Wiersema, K., "The Afterglows of Swift-era Gamma-Ray Bursts. II. Type I GRB versus Type II GRB Optical Afterglows," ApJ 734, 96 (June 2011).

[19] Li, L.-X. and Paczyński, B., "Transient Events from Neutron Star Mergers," ApJ 507, L59-L62 (Nov. 1998). 
[20] Metzger, B. D., Martínez-Pinedo, G., Darbha, S., Quataert, E., Arcones, A., Kasen, D., Thomas, R., Nugent, P., Panov, I. V., and Zinner, N. T., "Electromagnetic counterparts of compact object mergers powered by the radioactive decay of r-process nuclei," MNRAS 406, 2650-2662 (Aug. 2010).

[21] Barnes, J. and Kasen, D., "Effect of a High Opacity on the Light Curves of Radioactively Powered Transients from Compact Object Mergers," ApJ 775, 18 (Sept. 2013).

[22] Ott, C. D., "TOPICAL REVIEW: The gravitational-wave signature of core-collapse supernovae," Classical and Quantum Gravity 26, 063001 (Mar. 2009).

[23] Campana, S., Mangano, V., Blustin, A. J., Brown, P., Burrows, D. N., Chincarini, G., Cummings, J. R., Cusumano, G., Della Valle, M., Malesani, D., Mészáros, P., Nousek, J. A., Page, M., Sakamoto, T., Waxman, E., Zhang, B., Dai, Z. G., Gehrels, N., Immler, S., Marshall, F. E., Mason, K. O., Moretti, A., O’Brien, P. T., Osborne, J. P., Page, K. L., Romano, P., Roming, P. W. A., Tagliaferri, G., Cominsky, L. R., Giommi, P., Godet, O., Kennea, J. A., Krimm, H., Angelini, L., Barthelmy, S. D., Boyd, P. T., Palmer, D. M., Wells, A. A., and White, N. E., "The association of GRB 060218 with a supernova and the evolution of the shock wave," Nature 442, 1008-1010 (Aug. 2006).

[24] Schawinski, K., Justham, S., Wolf, C., Podsiadlowski, P., Sullivan, M., Steenbrugge, K. C., Bell, T., Röser, H.-J., Walker, E. S., Astier, P., Balam, D., Balland, C., Carlberg, R., Conley, A., Fouchez, D., Guy, J., Hardin, D., Hook, I., Howell, D. A., Pain, R., Perrett, K., Pritchet, C., Regnault, N., and Yi, S. K., "Supernova Shock Breakout from a Red Supergiant," Science 321, 223-226 (July 2008).

[25] Woosley, S. E. and Bloom, J. S., "The Supernova Gamma-Ray Burst Connection," ARA\&A 44, 507-556 (Sept. 2006).

[26] Leonor, I., Cadonati, L., Coccia, E., D’Antonio, S., Di Credico, A., Fafone, V., Frey, R., Fulgione, W., Katsavounidis, E., Ott, C. D., Pagliaroli, G., Scholberg, K., Thrane, E., and Vissani, F., "Searching for prompt signatures of nearby core-collapse supernovae by a joint analysis of neutrino and gravitational wave data," Classical and Quantum Gravity 27, 084019 (Apr. 2010).

[27] Siemens, X., Creighton, J., Maor, I., Majumder, S. R., Cannon, K., and Read, J., "Gravitational wave bursts from cosmic (super)strings: Quantitative analysis and constraints," Phys. Rev. D 73, 105001 (May 2006).

[28] Damour, T. and Vilenkin, A., "Gravitational Wave Bursts from Cosmic Strings," Physical Review Letters 85, 3761-3764 (Oct. 2000).

[29] Aasi, J., Abadie, J., Abbott, B. P., Abbott, R., Abbott, T., Abernathy, M. R., Accadia, T., Acernese, F., Adams, C., Adams, T., and et al., "Constraints on Cosmic Strings from the LIGO-Virgo Gravitational-Wave Detectors," Physical Review Letters 112, 131101 (Apr. 2014).

[30] Aasi, J., Abadie, J., Abbott, B. P., Abbott, R., Abbott, T., Abernathy, M. R., Accadia, T., Acernese, F., Adams, C., Adams, T., and et al., "First Searches for Optical Counterparts to Gravitational-wave Candidate Events," ApJS 211, 7 (Mar. 2014).

[31] Evans, P. A., Fridriksson, J. K., Gehrels, N., Homan, J., Osborne, J. P., Siegel, M., Beardmore, A., Handbauer, P., Gelbord, J., Kennea, J. A., and et al., "Swift Follow-up Observations of Candidate Gravitationalwave Transient Events," ApJS 203, 28 (Dec. 2012).

[32] Abadie, J., Abbott, B. P., Abbott, R., Abbott, T. D., Abernathy, M., Accadia, T., Acernese, F., Adams, C., Adhikari, R., Affeldt, C., and et al., "First low-latency LIGO+Virgo search for binary inspirals and their electromagnetic counterparts," A\&A 541, A155 (May 2012).

[33] LIGO Scientific Collaboration, Virgo Collaboration, Abadie, J., Abbott, B. P., Abbott, R., Abbott, T. D., Abernathy, M., Accadia, T., Acernese, F., Adams, C., and et al., "Implementation and testing of the first prompt search for gravitational wave transients with electromagnetic counterparts," A\&A 539, A124 (Apr. 2012).

[34] Gonzalez, G. and Vinet J.-Y. https://dcc.ligo.org/LIGO-M1300550/public.

[35] Gonzalez, G. and Vinet J.-Y. https://dcc.ligo.org/LIGO-F1300021/public.

[36] LIGO Scientific Collaboration, Virgo Collaboration, Aasi, J., Abadie, J., Abbott, B. P., Abbott, R., Abbott, T. D., Abernathy, M., Accadia, T., Acernese, F., and et al., "Prospects for Localization of Gravitational Wave Transients by the Advanced LIGO and Advanced Virgo Observatories," ArXiv e-prints (Apr. 2013). 
[37] Abbott, R., Adhikari, R., Ballmer, S., Barsotti, L., Evans, M., Fritschel, P., Frolov, V., Mueller, G., Slagmolen, B., and S., W., "Advanced ligo length sensing and control final design," (2010).

[38] Marion, F., "Multiband search of coalescing binaries applied to VIRGO CITF data," in [American Institute of Physics Conference Series], Proceedings of Rencontres de Moriond on Gravitational Waves and Experimental Gravity, 2003 (2004).

[39] Sathyaprakash, B. S. and Schutz, B. F., "Physics, Astrophysics and Cosmology with Gravitational Waves," Living Reviews in Relativity 12, 2 (Mar. 2009).

[40] Cannon, K., Cariou, R., Chapman, A., Crispin-Ortuzar, M., Fotopoulos, N., Frei, M., Hanna, C., Kara, E., Keppel, D., Liao, L., Privitera, S., Searle, A., Singer, L., and Weinstein, A., "Toward Early-warning Detection of Gravitational Waves from Compact Binary Coalescence," ApJ 748, 136 (Apr. 2012).

[41] Cannon, K., Hanna, C., and Keppel, D., "Efficiently enclosing the compact binary parameter space by singular-value decomposition," Phys. Rev. D 84, 084003 (Oct. 2011).

[42] Cannon, K., Chapman, A., Hanna, C., Keppel, D., Searle, A. C., and Weinstein, A. J., "Singular value decomposition applied to compact binary coalescence gravitational-wave signals," Phys. Rev. D 82, 044025 (Aug 2010).

[43] Allen, B., Anderson, W. G., Brady, P. R., Brown, D. A., and Creighton, J. D. E., "FINDCHIRP: An algorithm for detection of gravitational waves from inspiraling compact binaries," Phys. Rev. D 85, 122006 (June 2012).

[44] Cannon, K., Hanna, C., and Keppel, D., "Method to estimate the significance of coincident gravitationalwave observations from compact binary coalescence," Phys. Rev. D 88, 024025 (July 2013).

[45] Beauville, F., Bizouard, M.-A., Blackburn, L., Bosi, L., Brocco, L., Brown, D. A., Buskulic, D., Cavalier, F., Chatterji, S., Christensen, N., Clapson, A.-C., Fairhurst, S., Grosjean, D., Guidi, G., Hello, P., Heng, S., Hewitson, M., Katsavounidis, E., Klimenko, S., Knight, M., Lazzarini, A., Leroy, N., Marion, F., Markowitz, J., Melachrinos, C., Mours, B., Ricci, F., Viceré, A., Yakushin, I., Zanolin, M., and joint LIGO/Virgo working Group, "Detailed comparison of LIGO and Virgo inspiral pipelines in preparation for a joint search," Classical and Quantum Gravity 25, 045001 (Feb. 2008).

[46] Klimenko, S. and et al., "Constraint likelihood analysis for a network of gravitational wave detectors," Phys. Rev. D 72, 122002 (2005).

[47] Abadie, J. and et al., "All-sky search for gravitational-wave bursts in the first joint LIGO-GEO-Virgo run," Phys.Rev. D81, 102001 (2010).

[48] Abbott, B. P. and et al., "Search for gravitational-wave bursts in the first year of the fifth LIGO science run," Phys. Rev. D 80, 102001 (2009).

[49] GraCEDb. https://www.lsc-group.phys.uwm.edu/daswg/projects/gracedb.html.

[50] LVAlert. https://www.lsc-group.phys.uwm.edu/daswg/projects/lvalert.html.

[51] Fairhurst, S., "Triangulation of gravitational wave sources with a network of detectors," New J.Phys. 11, 123006 (2009).

[52] Singer, L. P., Price, L. R., Farr, B., Urban, A. L., Pankow, C., Vitale, S., Veitch, J., Farr, W. M., Hanna, C., Cannon, K., Downes, T., Graff, P., Haster, C.-J., Mandel, I., Sidery, T., and Vecchio, A., "The First Two Years of Electromagnetic Follow-Up with Advanced LIGO and Virgo," ArXiv e-prints (Apr. 2014).

[53] O'Shaughnessy, R., Kim, C., Fragos, T., Kalogera, V., and Belczynski, K., "Constraining Population Synthesis Models via the Binary Neutron Star Population," ApJ 633, 1076-1084 (Nov. 2005).

[54] Barthelmy, S. D., "The GCN web site," (2009). http://gcn.gsfc.nasa.gov/gcn/.

[55] Aasi, J., Abadie, J., Abbott, B. P., Abbott, R., Abbott, T. D., Abernathy, M., Accadia, T., Acernese, F., Adams, C., Adams, T., and et al., "Parameter estimation for compact binary coalescence signals with the first generation gravitational-wave detector network," Phys. Rev. D 88, 062001 (Sept. 2013).

[56] Vitale, S., Del Pozzo, W., Li, T. G. F., Van Den Broeck, C., Mandel, I., Aylott, B., and Veitch, J., "Effect of calibration errors on Bayesian parameter estimation for gravitational wave signals from inspiral binary systems in the advanced detectors era," Phys. Rev. D 85, 064034 (Mar. 2012). 
[57] Vitale, S., Del Pozzo, W., Li, T. G. F., Van Den Broeck, C., Aylott, B., and Veitch, J., "Effect of calibration errors on Bayesian parameter estimation for gravitational wave signals from inspiral binary systems in the advanced detectors era: Further investigations," Journal of Physics Conference Series 484, 012026 (Mar. 2014).

[58] Abadie, J., Abbott, B. P., Abbott, R., Abernathy, M., Accadia, T., Acernese, F., Adams, C., Adhikari, R., Ajith, P., Allen, B., and et al., "TOPICAL REVIEW: Predictions for the rates of compact binary coalescences observable by ground-based gravitational-wave detectors," Classical and Quantum Gravity 27, 173001 (Sept. 2010).

[59] Veitch, J., Mandel, I., Aylott, B., Farr, B., Raymond, V., Rodriguez, C., van der Sluys, M., Kalogera, V., and Vecchio, A., "Estimating parameters of coalescing compact binaries with proposed advanced detector networks," Phys. Rev. D 85, 104045 (May 2012).

[60] Fairhurst, S., "Source localization with an advanced gravitational wave detector network," Classical and Quantum Gravity 28, 105021 (May 2011).

[61] Vitale, S. and Zanolin, M., "Application of asymptotic expansions for maximum likelihood estimators' errors to gravitational waves from inspiraling binary systems: The network case," Phys. Rev. D 84, 104020 (Nov. 2011).

[62] Klimenko, S., Vedovato, G., Drago, M., Mazzolo, G., Mitselmakher, G., Pankow, C., Prodi, G., Re, V., Salemi, F., and Yakushin, I., "Localization of gravitational wave sources with networks of advanced detectors," Phys. Rev. D 83, 102001 (May 2011).

[63] Singer, L. and et al. http://www.ligo.org/science/first2years (2014).

[64] Canizares, P., Field, S. E., Gair, J. R., and Tiglio, M., "Gravitational wave parameter estimation with compressed likelihood evaluations," Phys. Rev. D 87, 124005 (June 2013).

[65] Smith, R., Hanna, C., Mandel, I., and Vecchio, A., "Rapidly evaluating the compact binary likelihood function via interpolation," ArXiv e-prints (May 2013). 